\title{
World Journal of Surgical Oncology reviewer acknowledgement 2014
}

Manoj Pandey ${ }^{1 *}$ and Thalyana Smith-Vikos ${ }^{2}$

\section{Contributing reviewers}

The editors of World Journal of Surgical Oncology would like to thank all our reviewers who have contributed to the journal in Volume 12 (2014).

Remzi Abali
Turkey

Mahmoud Abbas

Germany

Zakaria Abd Elmageed

United States of America

Essam Abdelalim

Qatar

Iman Abdelgawad

Egypt

Badr Abdull Gaffar

United Arab Emirates

Aswin George Abraham

United Kingdom

Pedro Acién

Spain

Pietro Addeo

France

Prasad Adusumilli

United States of America

Mehmet Agilli

Turkey

Sastha Ahanatha Pillai India

Hanaa Ahmed

Egypt
Sung-Ja Ahn

South Korea

Masaki Aizawa

Japan

Sami Akbulut

Turkey

Alper Akcan

Turkey

Ali Akdemir

Turkey

Margarete Akens

Canada

Ali Akhavan

Iran

Javed Akhtar

Pakistan

Md. Zahid Akhter

India

Takashi Akiyoshi

Japan

Matthew Albert

United States of America

Manuel Alcántara Moral Spain

Luca Antonio Aldrighetti Italy

\begin{abstract}
Abraham Alexander
Canada
\end{abstract}

George Alexiou

Greece

Abdullah Algharib

United Kingdom

Badar Alhadhrami

Oman

Bilal Al-Jiffry

Saudi Arabia

Eyas Alkhalili

United States of America

Marco Allaix

Italy

Asma AlNajjar

Saudi Arabia

Tahseen Al-Saleem

United States of America

Abdulmalik Altaf

Saudi Arabia

Giordano Alves

Brazil

Saadallah Al-Zacko

Iraq

Maria Raffaella Ambrosio

Italy

\footnotetext{
* Correspondence: wjso@biomedcentral.com

'Bhopal Memorial Hospital and Research Center, Bhanpur, Bhopal, Madhya

Pradesh, India
} 


\author{
Amedeo Amedei \\ Italy \\ Soheila Aminimoghaddam \\ Iran \\ Michele Ammendola \\ Italy \\ M. Robyn Andersen \\ United States of America \\ Andreas Andreou \\ Germany \\ Chaisiri Angkurawaranon \\ United Kingdom \\ Nagaraju Anreddy \\ United States of America \\ Myron Arlen \\ United States of America \\ Paul Arnold \\ United States of America \\ Grazia Artioli \\ Italy \\ Andreas Artopoulos \\ United Kingdom \\ Christopher Aston \\ United States of America \\ Maurice Efana Asuquo \\ Nigeria \\ Sam Atallah \\ United States of America \\ Riccardo A. Audisio \\ United Kingdom \\ Pirvu Augustin \\ France \\ Ana Maria Autran \\ Mexico \\ Aida Ayadi-Kaddour \\ Tunisia \\ Haruhuito Azuma \\ Japan \\ Woo Yong B Ae \\ South Korea \\ Hideo Baba \\ Japan \\ Mahwash Babar \\ United Kingdom
}

Sung Uk Bae

South Korea

Jae Hyun Bae

South Korea

Badrulhisham Bahadzor

Malaysia

Gopisankar Balaji

India

Andrzej Balcerowiak

Poland

Domingo Balderramo

Argentina

Elizabeth Baldini

United States of America

Mark Ball

United States of America

Jheelam Banerjee

United States of America

Tahmina Banu

Bangladesh

Nikolaos Barbetakis

Greece

John Barr

United States of America

Antonio Barrasa

Spain

Allison Barrett

United States of America

Stefano Basile

Italy

Stefano Basso

Italy

Rashmi Bathri

India

Marco Johannes Battista

Germany

Joel Baumgartner

United States of America

Daniel Baumunk

Germany

Daniel Becker

United States of America

Natalia Bejarano

Spain
Mehtap Beker Acay

Turkey

Seyda Belli

Turkey

Omar Bellorin

United States of America

Marcelo Beltran

Chile

Vladimír Benes

Czech Republic

Lothar Bergmann

Germany

Alejandro Bermudez-García

Peru

Emilio Bertani

Italy

Sergio Bertoglio

Italy

Luca Bertolaccini

Italy

Lorenzo Bianchi

Italy

Pierre Bigot

France

Vivek Bindal

India

Akwasi Boateng

United States of America

Francesco Boccardo

Italy

Giorgio Bogani

Italy

Martin Bohac

Slovakia

Ilka Boin

Brazil

Francesca Bolacchi

Italy

Eleni Bolanaki

Greece

Ronell Bologna-Molina

Mexico

Rodrigo Bonhin

Brazil 


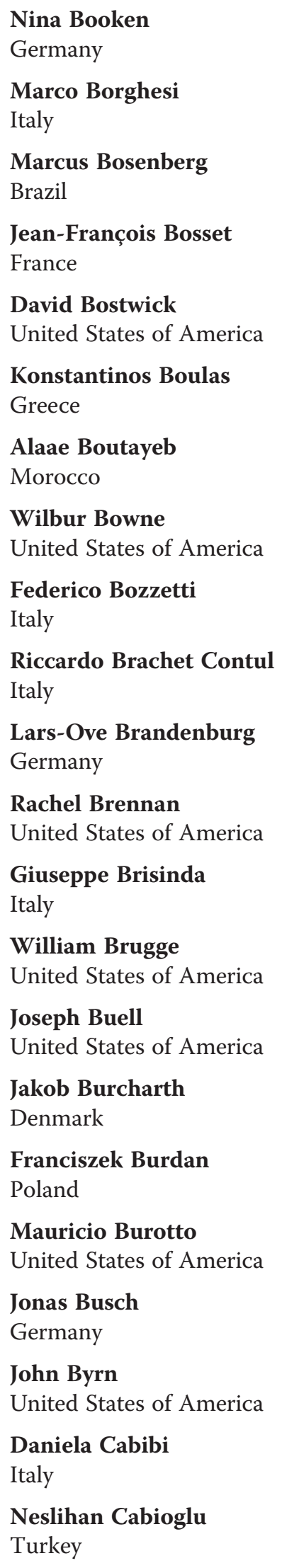

\begin{tabular}{|c|c|}
\hline $\begin{array}{l}\text { Francisco Cabrera-Mendoza } \\
\text { Mexico }\end{array}$ & $\begin{array}{l}\text { Irina-Draga Caruntu } \\
\text { Romania }\end{array}$ \\
\hline $\begin{array}{l}\text { Xiang-Ran Cai } \\
\text { China }\end{array}$ & $\begin{array}{l}\text { Marco Casaccia } \\
\text { Italy }\end{array}$ \\
\hline $\begin{array}{l}\text { Ana Laura Calderón-Garcidueñas } \\
\text { Mexico }\end{array}$ & $\begin{array}{l}\text { Riccardo Casadei } \\
\text { Italy }\end{array}$ \\
\hline $\begin{array}{l}\text { Pietro Caliandro } \\
\text { Italy }\end{array}$ & $\begin{array}{l}\text { Emanuele Castelli } \\
\text { Italy }\end{array}$ \\
\hline $\begin{array}{l}\text { Armando Calogero } \\
\text { Italy }\end{array}$ & $\begin{array}{l}\text { Giorgio Cattoretti } \\
\text { Italy }\end{array}$ \\
\hline $\begin{array}{l}\text { Juan Pablo Campana } \\
\text { Argentina }\end{array}$ & $\begin{array}{l}\text { Luigi Cavanna } \\
\text { Italy }\end{array}$ \\
\hline $\begin{array}{l}\text { Luca Campana } \\
\text { Italy }\end{array}$ & $\begin{array}{l}\text { Subrata Chakrabarti } \\
\text { United States of America }\end{array}$ \\
\hline $\begin{array}{l}\text { Fábio Campos } \\
\text { Brazil }\end{array}$ & $\begin{array}{l}\text { Sudipta Chakrabarti } \\
\text { India }\end{array}$ \\
\hline $\begin{array}{l}\text { Ferdinando Carlo Maria Cananzi } \\
\text { Italy }\end{array}$ & $\begin{array}{l}\text { Rubel Chakravarty } \\
\text { India }\end{array}$ \\
\hline $\begin{array}{l}\text { Aras Emre Canda } \\
\text { Turkey }\end{array}$ & $\begin{array}{l}\text { Stephen Chan } \\
\text { Hong Kong }\end{array}$ \\
\hline $\begin{array}{l}\text { Alberto Candau-Alvarez } \\
\text { Spain }\end{array}$ & $\begin{array}{l}\text { Wen-Wei Chang } \\
\text { Taiwan }\end{array}$ \\
\hline $\begin{array}{l}\text { Myrna Candelaria } \\
\text { Mexico }\end{array}$ & $\begin{array}{l}\text { Benoit Chaput } \\
\text { France }\end{array}$ \\
\hline $\begin{array}{l}\text { Francisco Candido dos Reis } \\
\text { Brazil }\end{array}$ & $\begin{array}{l}\text { Mary Charlton } \\
\text { United States of America }\end{array}$ \\
\hline $\begin{array}{l}\text { Edwin Canete } \\
\text { Philippines }\end{array}$ & $\begin{array}{l}\text { Mongkon Charoenpitakchai } \\
\text { Thailand }\end{array}$ \\
\hline $\begin{array}{l}\text { Robert Canter } \\
\text { United States of America }\end{array}$ & $\begin{array}{l}\text { Grigoris Chatzimavroudis } \\
\text { Greece }\end{array}$ \\
\hline $\begin{array}{l}\text { Hui Cao } \\
\text { China }\end{array}$ & $\begin{array}{l}\text { Bicheng Chen } \\
\text { China }\end{array}$ \\
\hline $\begin{array}{l}\text { Guangwen Cao } \\
\text { China }\end{array}$ & $\begin{array}{l}\text { Zhe-Sheng Chen } \\
\text { United States of America }\end{array}$ \\
\hline $\begin{array}{l}\text { Bangwei Cao } \\
\text { China }\end{array}$ & $\begin{array}{l}\text { Kaiyun Chen } \\
\text { China }\end{array}$ \\
\hline $\begin{array}{l}\text { Salvatore Cappabianca } \\
\text { Italy }\end{array}$ & $\begin{array}{l}\text { Xiaolei Chen } \\
\text { China }\end{array}$ \\
\hline $\begin{array}{l}\text { Sergio Carandina } \\
\text { France }\end{array}$ & $\begin{array}{l}\text { Jun Chen } \\
\text { China }\end{array}$ \\
\hline $\begin{array}{l}\text { Philip Carrott } \\
\text { United States of America }\end{array}$ & $\begin{array}{l}\text { Feng Chen } \\
\text { China }\end{array}$ \\
\hline $\begin{array}{l}\text { William Carson } \\
\text { United States of America }\end{array}$ & $\begin{array}{l}\text { Yongshun Chen } \\
\text { China }\end{array}$ \\
\hline
\end{tabular}


Zong-You Chen

China

Wen Cheng

China

Wah Cheuk

Hong Kong

Pan Chi

China

Tawona Chinembiri

South Africa

Hung-Yi Chiou

Taiwan

Luis Chiva

Spain

Min Chul Choi

South Korea

Rubens Chojniak

Brazil

Siu Ho Chok

Hong Kong

Siu Ho Kenneth Chok

China

Tam-Lin Chow

Hong Kong

Shouvik Chowdhury

India

Fei Chu

United States of America

Sheng-Hua Chu

China

Saulius Cicenas

Lithuania

Cara Cipriano

United States of America

Shannon Clark

United States of America

Paul Clarkson

Canada

Fabricio Coelho

Brazil

Marcela Pecora Cohen

Brazil

Riccardo Colombo

Italy

\author{
Alfredo Conti \\ Italy \\ Gennaro Cormio \\ Italy \\ Murat Cosar \\ Turkey \\ Carlos E Costa Almeida \\ Portugal \\ Vitor Costa Simoes \\ Portugal \\ Lisa Coviello \\ United States of America \\ Immacolata Cozzolino \\ Italy \\ Carmen Criscitiello \\ Italy \\ Ion Cristobal \\ Spain \\ Guanglin Cui \\ Norway \\ Andrew Currie \\ United Kingdom \\ Glenda da Silva \\ Brazil \\ Rikke Dahlrot \\ Denmark \\ Juncheng Dai \\ China \\ Alberto Dal Molin \\ Italy \\ Rolando M. D'Angelillo \\ Italy \\ Karina Danilowicz \\ Argentina \\ Alexander Daoud \\ United States of America \\ Christopher Dardis \\ United States of America \\ Guenter Daxenbichler \\ Austria \\ Remco de Bree \\ Netherlands \\ Juan R. de los Toyos \\ Spain
}

Giuseppe De Luca

Italy

Tarek Debs

France

Emil Dediol

Croatia

Yvonne Dei-Adomakoh

Ghana

Michela Del Prete

Italy

Silvana Del Vecchio

Italy

Marcos Dellaretti

Brazil

Andreas K Demetriades

United Kingdom

Hakan Demirci

Turkey

Ilhan Demirci

Germany

Hatice Mirac Binnaz Demirkan

Turkey

Chadrick Denlinger

United States of America

Chadrick Denlinger

United States of America

Nunzio Denora

Italy

Kristoffer Derwinger

Sweden

Daniel Deschler

United States of America

Jasreman Dhillon

United States of America

Francesco Di Costanzo

Italy

Roberto Di Donato

Italy

Alberto Di Leo

Italy

Massimo Di Maio

Italy

Bernardo Dias Pereira

Portugal 
Julio Diaz-Perez

United States of America

\section{Andrew Dickinson}

Canada

Claudius Diez

Germany

Dale Ding

United States of America

Xiangwu Ding

China

Marius Distler

Germany

Rosa Divella

Italy

Anthony Dixon

United Kingdom

Bozena Dobrzycka

Poland

Philipp Doebler

Germany

Xiaorong Dong

China

Zhou Donglei

China

Piotr Donizy

Poland

Efrat Dotan

United States of America

Dimitrios Dragoumis

United Kingdom

Lutao Du

China

Xiaohui Duan

China

Berna Bozkurt Duman

Turkey

Traian Dumitrascu

Romania

Edward Ebramzadeh

United States of America

Ovie Edafe

United Kingdom

Barish Edil

United States of America
Bijan Eghtesad

United States of America

Brian Egleston

United States of America

Günter Eisele

Switzerland

Christopher Ekwunife

Nigeria

Omar Elmalki

Morocco

Adel El-Naggar

United States of America

Ahmed Elzawawy

Egypt

Shigenobu Emoto

Japan

Giorgio Ercolani

Italy

Katerina Erokhina

United States of America

Emanuela Esposito

Italy

Jon Etxano

Spain

Laura Evangelista

Italy

Clarisse Eveno

France

René Fahrner

Germany

Mark Fairweather

United States of America

Khashayar Fakhrian

Germany

Nicolas Fakhry

France

Anna-Karin Falck

Sweden

Daniela Fanni

Italy

Walid Faraj

Lebanon

Sükösd Farkas

Hungary
Farshad Farshidfar

Canada

Giammarco Fava

Italy

Iain Feeley

Ireland

Yang Fei

China

Luigi Fenoglio

Italy

Clarissa Fernandes

Brazil

André Figueiredo

Brazil

Vittorio Fineschi

Italy

Enrico Fiori

Italy

Paul Fitzpatrick

Sweden

Rosario Fornaro

Italy

Dirk Forner

Germany

Simone Forterre

Switzerland

Lucio Fortunato

Italy

Caterina Fotia

Italy

Adam Enver Frampton

United Kingdom

Alessandro Franchello

Italy

Renato Franco

Italy

Marolleau François

Belgium

Richard K Freeman

United States of America

Deliang Fu

China

Takaaki Fujii

Japan 


\begin{tabular}{|c|c|c|}
\hline Shin Fujita & Hanspeter Gerber & Philip Gordon \\
\hline Japan & United States of America & Canada \\
\hline Takeo Fujita & Sarbani Ghosh Laskar & Mikel Gorostidi \\
\hline Japan & India & Spain \\
\hline Tetsuji Fujita & Alex Giakoustidis & Francois Gouin \\
\hline Japan & United Kingdom & France \\
\hline Tetsuo Fujita & Pierluigi Giampaolino & Cara Govednik \\
\hline Japan & Italy & United States of America \\
\hline Yoshinori Fujiwara & Alessandro Giardino & Claudia Gragnoli \\
\hline Japan & Italy & United States of America \\
\hline Yosuke Fukunaga & Joanna Gibson & Ewen Griffiths \\
\hline Japan & United States of America & United Kingdom \\
\hline Naotake Funamizu & Anthony Gill & Peter Grimminger \\
\hline Japan & Australia & Germany \\
\hline Philipp Funovics & Erika Ginsburg & Valentina Grossi \\
\hline Austria & United States of America & Italy \\
\hline Yoshitaka Furuya & Luca Giovanella & Christina Gruber \\
\hline Japan & Switzerland & Austria \\
\hline Csaba Gajdos & Sara Giovannoni & Thomas Grunewald \\
\hline United States of America & Italy & France \\
\hline Valerio Gallotta & Pier Giulianotti & Katharina Grupp \\
\hline Italy & United States of America & Germany \\
\hline Andrea Galosi & Gabriel Glockzin & Salvatore Gruttadauria \\
\hline Italy & Germany & Italy \\
\hline Moses Galukande & Paul Gobardhan & Yan hong Gu \\
\hline Uganda & Netherlands & China \\
\hline Sean Galvin & Sean Goh & Guoli Gu \\
\hline Australia & Australia & China \\
\hline Donatella Gambini & Ilhami Gok & Wenxian Guan \\
\hline Italy & Turkey & China \\
\hline Gerald Gantt, Jr. & Mónica Gomes & Wenxian Guan \\
\hline United States of America & Portugal & China \\
\hline Rebeca Garcia-Roman & Nelma Gomes & Maria Guembe \\
\hline Mexico & United Kingdom & Spain \\
\hline Jerad Gardner & Fernando Gómez & Francesco Guerra \\
\hline United States of America & Spain & Italy \\
\hline Sue Garnett & Li Gong & Wang Guiqi \\
\hline United Kingdom & China & China \\
\hline Louie Gaston & Weihua Gong & Dorothy Gujral \\
\hline United Kingdom & China & United Kingdom \\
\hline Florian Gebauer & Luis González & Antonino Gulino \\
\hline Germany & Spain & Italy \\
\hline Georgios Georgiou & David González-Flores & Serkan Guneyli \\
\hline Greece & Spain & Turkey \\
\hline
\end{tabular}




\begin{tabular}{|c|c|c|}
\hline $\begin{array}{l}\text { Shutaro Gunji } \\
\text { Japan }\end{array}$ & $\begin{array}{l}\text { Ayshamgul Hasim } \\
\text { China }\end{array}$ & $\begin{array}{l}\text { Miguel Hissa } \\
\text { Brazil }\end{array}$ \\
\hline $\begin{array}{l}\text { Gang Guo } \\
\text { China }\end{array}$ & $\begin{array}{l}\text { Eva Haspinger } \\
\text { Italy }\end{array}$ & $\begin{array}{l}\text { Ming-Chih Ho } \\
\text { Taiwan }\end{array}$ \\
\hline $\begin{array}{l}\text { Lihe Guo } \\
\text { China }\end{array}$ & $\begin{array}{l}\text { Masaharu Hata } \\
\text { Japan }\end{array}$ & $\begin{array}{l}\text { Thomas Höfner } \\
\text { Germany }\end{array}$ \\
\hline $\begin{array}{l}\text { Yi Guo } \\
\text { China }\end{array}$ & $\begin{array}{l}\text { Etsuro Hatano } \\
\text { Japan }\end{array}$ & $\begin{array}{l}\text { Konstanze Holl-Ulrich } \\
\text { Germany }\end{array}$ \\
\hline $\begin{array}{l}\text { Ketai Guo } \\
\text { Germany }\end{array}$ & $\begin{array}{l}\text { Ali Can Hatemi } \\
\text { Turkey }\end{array}$ & $\begin{array}{l}\text { Hedieh Honarpisheh } \\
\text { United States of America }\end{array}$ \\
\hline $\begin{array}{l}\text { Shailvi Gupta } \\
\text { United States of America }\end{array}$ & $\begin{array}{l}\text { Peter Hauser } \\
\text { Hungary }\end{array}$ & $\begin{array}{l}\text { Simon Horenblas } \\
\text { Netherlands }\end{array}$ \\
\hline $\begin{array}{l}\text { Simona Gurzu } \\
\text { Romania }\end{array}$ & $\begin{array}{l}\text { Brian Hayes } \\
\text { Ireland }\end{array}$ & $\begin{array}{l}\text { Jun Horiguchi } \\
\text { Japan }\end{array}$ \\
\hline $\begin{array}{l}\text { David Gyorki } \\
\text { Australia }\end{array}$ & $\begin{array}{l}\text { Mutlu Hayran } \\
\text { Turkey }\end{array}$ & $\begin{array}{l}\text { Karoline Horisberger } \\
\text { Germany }\end{array}$ \\
\hline $\begin{array}{l}\text { Alina Habic } \\
\text { Romania }\end{array}$ & $\begin{array}{l}\text { Shoichi Hazama } \\
\text { Japan }\end{array}$ & $\begin{array}{l}\text { Charlotte Höybye } \\
\text { Sweden }\end{array}$ \\
\hline $\begin{array}{l}\text { Angelita Habr-Gama } \\
\text { Brazil }\end{array}$ & $\begin{array}{l}\text { Hong He } \\
\text { Australia }\end{array}$ & $\begin{array}{l}\text { Bo-Yuan Hsiao } \\
\text { Taiwan }\end{array}$ \\
\hline $\begin{array}{l}\text { Vijay Hadda } \\
\text { India }\end{array}$ & $\begin{array}{l}\text { Ashraf Hefny } \\
\text { Egypt }\end{array}$ & $\begin{array}{l}\text { Chih-Cheng Hsieh } \\
\text { Taiwan }\end{array}$ \\
\hline $\begin{array}{l}\text { Tetsuo Hagino } \\
\text { Japan }\end{array}$ & $\begin{array}{l}\text { Baukje Hemmes } \\
\text { Netherlands }\end{array}$ & $\begin{array}{l}\text { Meng Chiao Hsieh } \\
\text { Taiwan }\end{array}$ \\
\hline $\begin{array}{l}\text { Oliver Hakenberg } \\
\text { Germany }\end{array}$ & $\begin{array}{l}\text { Adam Hermanowicz } \\
\text { Poland }\end{array}$ & $\begin{array}{l}\text { Min Shu Hsieh } \\
\text { Taiwan }\end{array}$ \\
\hline $\begin{array}{l}\text { Geoffrey Hallock } \\
\text { United States of America }\end{array}$ & $\begin{array}{l}\text { Adrian Hibberd } \\
\text { Australia }\end{array}$ & $\begin{array}{l}\text { Chien-Jen Hsu } \\
\text { Taiwan }\end{array}$ \\
\hline $\begin{array}{l}\text { Madoka Hamada } \\
\text { Japan }\end{array}$ & $\begin{array}{l}\text { Shigeaki Higashiyama } \\
\text { Japan }\end{array}$ & $\begin{array}{l}\text { Jian-Kun Hu } \\
\text { China }\end{array}$ \\
\hline $\begin{array}{l}\text { Elham Hamed } \\
\text { Egypt }\end{array}$ & $\begin{array}{l}\text { Jens Hillengass } \\
\text { Germany }\end{array}$ & $\begin{array}{l}\text { Yingqi Hua } \\
\text { China }\end{array}$ \\
\hline $\begin{array}{l}\text { Ho-Seong Han } \\
\text { South Korea }\end{array}$ & $\begin{array}{l}\text { Tomoko Hirakata } \\
\text { Japan }\end{array}$ & $\begin{array}{l}\text { Dong Hua } \\
\text { China }\end{array}$ \\
\hline $\begin{array}{l}\text { Ping Han } \\
\text { China }\end{array}$ & $\begin{array}{l}\text { Daisaku Hirano } \\
\text { Japan }\end{array}$ & $\begin{array}{l}\text { Chao-Yuan Huang } \\
\text { Taiwan }\end{array}$ \\
\hline $\begin{array}{l}\text { Christopher Hancock } \\
\text { United States of America }\end{array}$ & $\begin{array}{l}\text { Satoshi Hirano } \\
\text { Japan }\end{array}$ & $\begin{array}{l}\text { Ling Huang } \\
\text { China }\end{array}$ \\
\hline $\begin{array}{l}\text { Ding-Jun Hao } \\
\text { China }\end{array}$ & $\begin{array}{l}\text { Yosuke Hirasawa } \\
\text { Japan }\end{array}$ & $\begin{array}{l}\text { Jing Huang } \\
\text { China }\end{array}$ \\
\hline $\begin{array}{l}\text { Saroona Haroon } \\
\text { Pakistan }\end{array}$ & $\begin{array}{l}\text { Masahiko Hirota } \\
\text { Japan }\end{array}$ & $\begin{array}{l}\text { Chao-Hung Hung } \\
\text { Taiwan }\end{array}$ \\
\hline $\begin{array}{l}\text { Yasuhisa Hasegawa } \\
\text { Japan }\end{array}$ & $\begin{array}{l}\text { Takeshi Hisa } \\
\text { Japan }\end{array}$ & $\begin{array}{l}\text { Soo Young Hur } \\
\text { South Korea }\end{array}$ \\
\hline
\end{tabular}


Sang Hwang

Australia

Maurizio Iacobone

Italy

Toni Ibrahim

Italy

Kenneth Iczkowski

United States of America

Benedetto Ielpo

Spain

Tsukasa Ikeura

Japan

Hisashi Ikoma

Japan

Kadri Ila

Turkey

Ray Iles

United Kingdom

Teruo Inamoto

Japan

Masafumi Inomata

Japan

Kentaro Inoue

Japan

Francesco Inzirillo

Italy

Orestis Ioannidis

Greece

Corey Iqbal

United States of America

Kamyar Iravani

Iran

Claudio Isella

Italy

Bjorn Isfoss

Norway

Hironori Ishihara

Japan

Takamichi Ishii

Japan

Arda Isik

Turkey

Fumiaki Isohashi

Japan
Yasuhiro Ito

Japan

Hideaki Ito

Japan

Shinji Itoh

Japan

Jakob Izbicki

Germany

Ira Jacobs

United States of America

Rana Jahanban-Esfahlan

Iran

Sumita Jain

India

James P Tam

Singapore

Fualal Jane Odubu

Uganda

Tae Jung Jang

South Korea

Tina Jaskoll

United States of America

Wojciech Jelski

Poland

Joon Jeong

South Korea

Hichem Jerraya

Tunisia

Bing JI

China

Shi-Wen Jiang

United States of America

Yong Jiang

United States of America

Chen Jin

China

Jie Jin

China

Tianbo Jin

China

Yinghua Jin

China

Jae-Won Joh

South Korea
Chris Jones

United Kingdom

Robin Jones

United States of America

Jean-Marc Joseph

Switzerland

Horng-Heng Juang

Taiwan

Mi Jun

China

Tareq Juratli

Germany

Vladimir Jurisic

Serbia

Elzbieta Kaczmarek

Poland

Ewa Kalinka-Warzocha

Poland

Kalpana Kalyanasundaram

Bhanumathy

Canada

Erdinc Kamer

Turkey

Osamu Kamihira

Japan

Toshiya Kamiyama

Japan

De-Zhi Kang

China

Harsh Kanhere

Australia

Fatih Karadeniz

South Korea

Elias Karakas

Germany

Selda Karamil

Turkey

Thedoros Karantanos

United States of America

Bernd Kasper

Germany

Venkata Katabathina

United States of America 
Rishil Kathawala

United States of America

Jaspreet Kaur

India

Shigeyuki Kawachi

Japan

Hisato Kawakami

Japan

Sakineh Kazemi Noureini

Iran

Mehmet Kefeli

Turkey

Edward Kendall

Canada

Mohammad Reza Keramati

Iran

Thomas Kerkhofs

Netherlands

Ugur KESICI

Turkey

Sonja Kessler

Germany

Akbar Khan

Canada

Hafiz Khan

United States of America

Piya Kiatisevi

Thailand

Yuko Kijima

Japan

Talat Kilic

Turkey

Min Joo Kim

South Korea

Jung Han Kim

South Korea

Hyun-Soo Kim

South Korea

Kyung Keun Kim

South Korea

Seong Hoon Kim

South Korea

Ji Eun Kim

South Korea
Soo Mi Kim

South Korea

Yasue Kimura

Japan

Johanna Kirchberg

Germany

Lawrence Kirschner

United States of America

Kinga Kiszka

Poland

Masahiro Kitada

Japan

Joji Kitayama

Japan

Jurgen Knuth

Germany

Shogo Kobayashi

Japan

Canan Kocaoglu

Turkey

Celalettin Kocaturk

Turkey

Bülent Koçer

Turkey

Ina Koch

Germany

Suman Kochhar

India

Takahiro Kogawa

United States of America

Naohiko Koide

Japan

Mahmut kuntay Kokanali

Turkey

Ender Koktekir

Turkey

Likurgos Kolilekas

Greece

Andrzej Komorowski

Poland

Tsunenori Kondo

Japan

Marina Kontogiorgi

Greece
Michael Kontos

United Kingdom

Piotr Korczynski

Poland

Ceren Korkmaz

Turkey

Ozge Korkmaz

Turkey

Ioannis Kostakis

Greece

Shin-ichi Kosugi

Japan

Christoforos Kotoulas

Greece

Andromachi Kougioumtzopoulou

Greece

Georgios Koutalellis

Greece

Mario Kramer

Germany

Avdyl Krasniqi

Serbia

Marco Krengli

Italy

Arvind Krishnamurthy

India

Naoshi Kubo

Japan

Naoshi Kubo

Japan

Harun Kucuk

Turkey

Ashok Kumar

India

Vinita Kumar

India

Vijay Kumar

India

Ramaiah Kumar

India

Yogesh Kumar

United States of America

Chikara Kunisaki

Japan 


\begin{tabular}{|c|c|c|}
\hline Jolanta Kupryjanczyk & Souhil Lebdai & Libo Liang \\
\hline Poland & France & China \\
\hline Sedef Kuran & Taek-Gu Lee & Ting-bo Liang \\
\hline Turkey & South Korea & China \\
\hline Atsushi Kurata & Yoon-Suk Lee & Myong Cheol Lim \\
\hline Japan & South Korea & South Korea \\
\hline Hiroaki Kuroda & Andreas Leithner & Sey Kiat Lim \\
\hline Japan & Austria & Singapore \\
\hline Katarzyna Kusnierz & Jose Leone & Michael Lim \\
\hline Poland & United States of America & United Kingdom \\
\hline Aamet Kuvat & Giovanni Leuzzi & Che Lin \\
\hline Turkey & Italy & Taiwan \\
\hline Nisreen Kweider & Valerae Lewis & Jules Lin \\
\hline Germany & United States of America & United States of America \\
\hline Stefano La Rosa & Chung-Pin Li & Yuchang Lin \\
\hline Italy & Taiwan & China \\
\hline Marco La Torre & Guoxin Li & Daniele Lindemann \\
\hline Italy & China & Brazil \\
\hline Jean Lachowicz & Yi Li & Chen Ling \\
\hline United States of America & China & United States of America \\
\hline Chao-Han Lai & Yong Li & Zhi-Qiang Ling \\
\hline Taiwan & China & China \\
\hline Jin-Yao Lai & Zhiyuan LI & Marcelo Linhares \\
\hline Taiwan & China & Brazil \\
\hline Silvia Lai & Bin Li & Caigang Liu \\
\hline Italy & United States of America & China \\
\hline Vinoth Kumar Lakshmanan & Jiangchao Li & Zhongchao Liu \\
\hline India & China & China \\
\hline Matteo Landriscina & Linfeng Li & Mu-Tai Liu \\
\hline Italy & United States of America & Taiwan \\
\hline Brian Lang & Yan Li & Min Liu \\
\hline Hong Kong & China & China \\
\hline Cord Langner & Yumin Li & Fenglin Liu \\
\hline Austria & China & China \\
\hline Anders Christian Larsen & Xinxiang Li & Qingquan Liu \\
\hline Denmark & China & China \\
\hline Konstantinos Lasithiotakis & Zhenzhen Li & Qing-Xin Liu \\
\hline Greece & China & China \\
\hline Johanna Laukkarinen & Enmin Li & Tang Liu \\
\hline Finland & China & China \\
\hline Daniela Lazar & Xiaoming Li & Zhenzhen Liu \\
\hline Romania & China & China \\
\hline Andreas Lazaris & Du Lianfang & Jing Liu \\
\hline Greece & China & China \\
\hline
\end{tabular}


Song-Mei Liu

China

Zeng-Shan Liu

China

Lorenzo Livi

Italy

Raúl Loera Valencia

Mexico

Varut Lohsiriwat

Thailand

Tommaso Lombardi

Switzerland

Francesco Loria

Italy

Karen Lounsbury

United States of America

Peter Lovrics

Canada

Veronica Loy

United States of America

Xueguan Lu

China

Guillaume Luc

France

Giuseppe Lucarelli

Italy

Jian Luo

China

Xiaofeng Lv

China

Elena Maccaroni

Italy

João Machado-Neto

Brazil

Antonio Macrì

Italy

Annette Maczurek

Australia

Marcelo Madeira

Brazil

Yoshihiko Maehara

Japan

Gaetano Magro

Italy
Haider Mahdi

United States of America

Ashish Mahendra

United Kingdom

Aditya Maheshwari

United States of America

Haroon Majeed

United Kingdom

Tafadzwa Makarawo

United States of America

Masujiro Makita

Japan

Antti Mäkitie

Finland

Zahra Maleki

United States of America

Supriya Mallick

India

Veronika Mancikova

Spain

Antonio Manenti

Italy

Giuditta Mannelli

Italy

James Manson

United Kingdom

Nicoletta Maounis

Greece

Makia Marafie

Kuwait

Luigi Marano

Italy

Ilaria Marech

Italy

Francesco Saverio

Mari Italy

Luigi Mariani

Italy

Ugo Marone

Italy

Daniele Marrelli

Italy

Robert Martin

United States of America
Carlos Martinez

Brazil

Vicenç Martinez Vecina

Andorra

Wojciech Marusza

Poland

Satoshi Maruyama

Japan

Salvatore Andrea Mastrolia

Israel

Norikazu Masuda

Japan

Toshihiko Masui

Japan

Leandro Matos

Brazil

Paraskevi Matsota

Greece

Junichi Matsui

Japan

Akiyo Matsumoto

Japan

Kazumasa Matsumoto

Japan

Kazuhiro Matsumoto

United States of America

Yoshihiro Matsumoto

Japan

Hiroshi Matsushita

Japan

Tobias Mattei

United States of America

Tobias Mattei

United States of America

Susumu Matuskuma

Japan

Tobias Maurer

Germany

Andreas Mavrogenis

Greece

Yarrow McConnell

Canada

Mabula Mchembe

Tanzania 
Jane McHowat

United States of America

Michael McIsaac

Canada

Fabio Medas

Italy

Fabio Medas

Italy

Aurora Medina-Sanson

Mexico

Meredith Medway

Australia

Diane Mege

France

Vinay Mehendale

India

Reza Mehrazin

United States of America

Jiong Mei

China

Mohamed Mekky

Egypt

Mostafa Melake

Egypt

Giulio Melloni

Italy

Muhammed Ashraf Memon

Australia

Fabio Mendes

Brazil

Fabrice Menegaux

France

Lourdes Mengual

Spain

Rudolf Mennigen

Germany

Francesca Merchionne

Italy

Walter Merkle

Germany

Roman Mezencev

United States of America

Nickolaos Michalopoulos

Greece
Anna Michno

Poland

Michal Mik

Poland

Milos Milosavljevic

Serbia

Ryogo Minamimoto

Japan

Bai Minghua

China

Olivier Mir

France

Vincenzo Mirone

Italy

Ali Mirsadeghi

Iran

Pradyumna Mishra

India

Koh Miura

Japan

Nobuyoshi MIyajima

Japan

Yasuyoshi Miyata

Japan

Toru Mizuguchi

Japan

Shugo Mizuno

Japan

Erito Mochiki

Japan

Sharon Moeno

South Africa

Rohit Moharil

India

Daniela Molena

United States of America

Adrian Molnar

Romania

Jennifer Monk

Canada

Katherine Moore

Canada

Luca Morelli

Italy
Mauricio Moreno

United States of America

Tasiuke Mori

Japan

Adriko Moses

Uganda

Mirna Mourtada-Maarabouni

United Kingdom

Dillip Muduly

India

Andre Mueller

United States of America

Oliver Mueller

Germany

Hetron Mweemba Munang'andu Norway

Miguel Muñoz

Spain

Tomohiro Murakawa

Japan

Atsuhiko Murata

Japan

Adrian Murillo

Spain

Michael Murphy

United States of America

Domenico Murrone

Italy

Nora Mutalima

United Kingdom

Dattatraya Muzumdar

India

Ospan Mynbaev

Russian Federation

Karuppiah Nagaraj

India

Mitsutoshi Nakada

Japan

Naoki Nakagawa

Japan

Ryota Nakamura

Japan

Tomoki Nakamura

Japan 


\author{
Satoshi Nakasu \\ Japan \\ Faina Nakhlis \\ United States of America \\ Tsutomu Namikawa \\ Japan
}

Nisana Namwat

Thailand

Moujhuri Nandi

India

Kannan Nar

India

Rajeswari Narayanappa

India

Masato Narita

Japan

Ofer Nativ

Israel

Pierina Navarria

Italy

Diana Navas Carrillo

Spain

Fred Nelson

United States of America

Barbara Netto

Brazil

Beng Kwang Ng

Malaysia

Rosa Nguyen

United States of America

Ourania Nicolatou-Galitis

Greece

Patricia Switten Nielsen

Denmark

Giuseppe Nigri

Italy

Yuji Nimura

Japan

Chunyu Niu

China

Xiaohui Niu

China

Takeo Nomi

Japan
Naohiro Nose

Japan

Markus Nottrott

Germany

Mohamed Nouh

Egypt

Alexander Novotny

Germany

Masahiro Nozawa

Japan

Achilleas Ntinas

Greece

Dimitrios Ntourakis

France

Ab Mutalib Nurul-Syakima

Malaysia

Daniel Nussbaum

United States of America

Chike Nzerue

United States of America

Toru Obuchi

Japan

Oliver O'Donovan

United Kingdom

Onder Ofluoglu

United States of America

Tomoko Ogawa

Japan

Yoshikazu Ogawa

Japan

Takao Ohtsuka

Japan

Hiroshi Okabe

Japan

Jiro Okami

Japan

Shinji Okano

Japan

Eiji Oki

Japan

Yoshinaga Okugawa

Japan

Scott Okuno

United States of America
Mana Oloomi

Iran

Georg Omlor

Germany

Ramesh Omranipour

Iran

Neamat Osman

Egypt

Naima Otmani

Morocco

Penelope Ottewell

United Kingdom

Anders Øverby

Norway

Jacqueline Oxenberg

United States of America

Seda Ozbek

Turkey

Irem Hicran Ozbudak

Turkey

Engin Ozcivici

Turkey

Salih Erpulat Ozis

Turkey

Safak Ozturk

Turkey

Furio Pacini

Italy

Somanath Padhi

India

Vincenzo Pagliarulo

Italy

Rama Pai

United States of America

Channing Paller

United States of America

Anshuman Pandey

India

Peter Panhofer

Austria

Yves Panis

France

Alberto Pansadoro

Italy 
Salil Kumar Parida

India

Alexander Parikh

United States of America

Dhavan Parikh

United States of America

Do Joong Park

South Korea

Joon Seong Park

South Korea

In Hae Park

South Korea

Toshima Parris

Sweden

Dinah Parums

United Kingdom

Huillaume Passot

France

Vita Pasukoniene

Lithuania

Atish Patel

United States of America

Mayur Patel

India

Ramnik Patel

United Kingdom

Sapna Patel

United States of America

Byomokesh Patro

India

Kitty Pavlakis

Greece

Luigi Michele Pavone

Italy

Jennifer Payne

Canada

Irene Pecorella

Italy

Gong Peng

China

Guang Peng

United States of America

Hanwei Peng

China
Ruiyun Peng

China

Sharaf Karim Perdawood

Denmark

Daniel Perez

Germany

Carles Pericay

Spain

Sebastien Perreault

Canada

Hitesh Peshavariya

Australia

Laura Peterson

United States of America

Marco Petrillo

Italy

Federico Piccioni

Italy

Gaetano Piccolo

Italy

Eugene Pietzak

United States of America

Eugene Pietzak

United States of America

Alessandro Pileri

Italy

Antonio Pinto

Italy

Georgios Pitoulias

Greece

Jan Plock

Switzerland

Vlad Porumb

Romania

Ignasi Poves

Spain

Tomasz Powrózek

Poland

Heru Pradjatmo

Indonesia

Adriano Massimiliano Priola

Italy

Giuseppe Procopio

Italy
Georgios Psychogios

Germany

Shilpa Puli

United States of America

Thipachart Punyaratabandhu

Thailand

Sumyra Qadri

India

Shadi Qasem

United States of America

Aamer Qazi

Canada

Xing Qi

China

Zhan Qiang

China

Zhiqiang Qin

United States of America

Silvia Quadrelli

Argentina

Sajida Qureshi

Pakistan

Marius Raica

Romania

Janusz Rak

Canada

Estela Regina Ramos Figueira

Brazil

Suresh Rana

United States of America

Federica Rascio

Italy

Edward Ratovitski

United States of America

Deepak Rautray

India

John-David Rebibo

France

Rishindra Reddy

United States of America

Jeremy Rees

United Kingdom

Jean Marc Regimbeau

France 


\begin{tabular}{|c|c|c|}
\hline Moacyr Rêgo & Giulio Rossi & Mirna Saldívar \\
\hline Brazil & Italy & Mexico \\
\hline Chandrababu Rejeeth & Valentina Rossi & Nikolaos Salemis \\
\hline India & Italy & Greece \\
\hline Bharat Rekhi & Rodnei Dennis Rossoni & Milan Samardjiski \\
\hline India & Brazil & Macedonia \\
\hline Song Ren & Krzysztof Roszkowski & Paolo sammartino \\
\hline China & Poland & Italy \\
\hline Zefang Ren & Steven Rothenberg & Juan Sancho \\
\hline China & United States of America & Spain \\
\hline Vivian Resende & Kenneth Rothfield & Michele Santangelo \\
\hline Brazil & United States of America & Italy \\
\hline Juan Antonio Retamero Diaz & Giuseppe Rubini & Matteo Santoni \\
\hline Spain & Italy & Italy \\
\hline Lyndsay Rhodes & Hannes Rudiger & Gouranga Santra \\
\hline United States of America & Switzerland & India \\
\hline Karl Riabowol & Juan Ruiz-García & Akira Sasaki \\
\hline Canada & Spain & Japan \\
\hline Riccardo Ricci & Maria Russell & Yusuke Sato \\
\hline Italy & United States of America & Japan \\
\hline Carina Riediger & Antonio Russo & Caroline Saucier \\
\hline Germany & Italy & Canada \\
\hline Luigi Rigacci & Giorgio Ivan Russo & Nadia Sawicka-Gutaj \\
\hline Italy & Italy & Poland \\
\hline Philipp Riss & Min-Hee Ryu & Stefano Scabini \\
\hline Austria & South Korea & Italy \\
\hline Rodolfo Rivas & Anton Sabashnikov & Peter Schemmer \\
\hline Mexico & United Kingdom & Germany \\
\hline Cleo Robinson & Feridoun Sabzi & Claus Schildberg \\
\hline Australia & Iran & Germany \\
\hline Nathan Robison & Lamiss Sad & Maximilian Schmeding \\
\hline United States of America & Egypt & Germany \\
\hline Jonathan Rodrigues & Tania Saibene & Moritz Schmelzle \\
\hline United Kingdom & Italy & Germany \\
\hline Mario A. Rodriguez & Leonard Saiegh & Carl Schmidt \\
\hline Mexico & Israel & United States of America \\
\hline José Antonio Rodríguez-Montes & Akio Sakamoto & Maren Schulze \\
\hline Spain & Japan & Germany \\
\hline Jong-Lyel Roh & Alborz Salavati & Roderich Schwarz \\
\hline South Korea & Iran & United States of America \\
\hline Andrea Romano & Alejandro Salazar & Roman Sefr \\
\hline Italy & Spain & Czech Republic \\
\hline Paul Rooney & Juan Antonio Salceda & Masau Sekiguchi \\
\hline United Kingdom & Argentina & Japan \\
\hline
\end{tabular}


Jayastu Senapati

India

Sanjeewa Seneviratne

Sri Lanka

Charalampos Seretis

Greece

Raffaele Serra

Italy

Sameh Sersar

Egypt

Monjri Shah

United States of America

Irshad Shaikh

United Kingdom

Guogen Shan

United States of America

Xiaobin Shang

China

Kiran Sharma

Canada

Daya Nand Sharma

India

Lizong Shen

China

Shun Shen

China

Victoria Shender

Russian Federation

Santosh Shenoy

United States of America

Gwo Tarng Sheu

Taiwan

Debing Shi

China

Jianhong Shi

China

Choong Nam Shim

South Korea

Kazuya Shinmura

Japan

Masahiro Shinoda

Japan

Akio Shiomi

Japan
Satoshi Shiono

Japan

Takkashi Shirobe

Japan

Vikas Sikri

India

Sabrina Silva

Brazil

Joao Manoel Silva Jr

Brazil

Nicola Silvestris

Italy

Nektaria Simiantonaki

Germany

Ronald Simon

Germany

Giuseppe Simone

Italy

Brijendra Singh

India

Cassian Sitaru

Germany

Ioannis Skandalos

Greece

Ayhan Sogut

Turkey

Leonardo Solaini

Italy

Olesya Solheim

Norway

Mamoon Solkar

United Kingdom

Chengli Song

China

Xicheng Song

China

Akshay Sood

United States of America

John Souglakos

Greece

Tutku Soyer

Turkey

Jens Sperling

Germany
Lucia Speroni

United States of America

Salvatore Squillaci

Italy

Anurag Srivastava

India

Katarzyna Starska

Poland

Philipp Storz-Pfennig

Germany

Russell Stothard

United Kingdom

Arne Streitbuerger

Germany

Philipp Ströbel

Germany

Moritz J Strowitzki

Germany

Tung-Hung Su

Taiwan

Takeki Sugimoto

Japan

Haruhiko Sugimura

Japan

Kiminori Sugino

Japan

Laurent Sulpice

France

Yang Sun

United States of America

Tong-Wen Sun

China

Wan-jun Sun

China

Simona Surdu

United States of America

Alexey Surov

Germany

Leslie Sutherland

Canada

Umesalma Syed

India

Isabella Syring

Germany 
Marcella Szuecs

Germany

Sabina Tabaczar

United Kingdom

Alphonse Taghian

United States of America

Saeed Taheri

Iran

Wanyi Tai

United States of America

Takeshi Takahara

Japan

Tadao Takano

Japan

Shigeo Takebayashi

Japan

Mitsuko Takenaga

Japan

Kengo Takeuchi

Japan

Shigeyuki Tamura

Japan

Marcus Tan

United States of America

Lijie Tan

China

Yasuyuki Tanahashi

Japan

Kuniya Tanaka

Japan

Eiji Tanaka

Japan

Torgrim Tandstad

Norway

Kai-Fu Tang

China

Nobuhiko Tanigawa

Japan

Shinya Tanimura

Japan

Junko Tanizaki

United States of America

Ozgur Tanriverdi

Turkey
Tawee Tanvetyanon

United States of America

Yungan Tao

France

Syed Tariq

United Kingdom

Muhammad Tariq

Pakistan

Adriana Tavares

Portugal

Ahmed Tawfik

Egypt

Giovanni Domenico Tebala

Isle of Man

Mohamedtaki Tejani

United States of America

Onur Telli

Turkey

Chris Terhaard

Netherlands

Evangelos Terpos

Greece

Ugo Testa

Italy

Cagatay Tezel

Turkey

Serdar Tezelman

Turkey

Hardik Thakker

India

Binay Thakur

Nepal

Bridie Thompson

Australia

Henrik Thorlacius

Sweden

Ole Thorlacius-Ussing

Denmark

Shifu Tian

United States of America

Mei Tian

China

Yuanhu Tian

China
Yanmei Tie

United States of America

Xavier Tillou

France

Andreas Toepfer

Germany

Gabriele Toietta

Italy

Yuji Toiyama

Japan

Kazuaki Tokodai

Japan

Yukio Tokumitsu

Japan

Jeffrey Tomaszewski

United States of America

Erkan Topkan

Turkey

William Tormey

Ireland

Alfonso Torquati

United States of America

Valerie Touitou

France

Edward Tredget

Canada

Giorgio Treglia

Switzerland

Matthew Trendowski

United States of America

Anna Truini

Italy

Stavros Tryfon

Greece

Gary Tse

Hong Kong

Nikolaos Tsoukalas

Greece

Takayuki Tsuchiya

United States of America

Hiroyuki Tsuchiya

Japan

Masumi Tsujiwaki

Japan 


\begin{tabular}{|c|c|c|}
\hline $\begin{array}{l}\text { Nelson Hirokazu Tsuno } \\
\text { Japan }\end{array}$ & $\begin{array}{l}\text { Giovanni Vennarecci } \\
\text { Italy }\end{array}$ & $\begin{array}{l}\text { Jin Wang } \\
\text { China }\end{array}$ \\
\hline $\begin{array}{l}\text { Shigeru Tsunoda } \\
\text { Japan }\end{array}$ & $\begin{array}{l}\text { Marilena Vered } \\
\text { Israel }\end{array}$ & $\begin{array}{l}\text { Jianhua Wang } \\
\text { China }\end{array}$ \\
\hline $\begin{array}{l}\text { Thomas } \mathbf{T u} \\
\text { Australia }\end{array}$ & $\begin{array}{l}\text { Sebastien Vergez } \\
\text { France }\end{array}$ & $\begin{array}{l}\text { Kun Wang } \\
\text { China }\end{array}$ \\
\hline $\begin{array}{l}\text { Kangsheng } \mathbf{T u} \\
\text { China }\end{array}$ & $\begin{array}{l}\text { Helene Verheije } \\
\text { Netherlands }\end{array}$ & $\begin{array}{l}\text { Qifeng Wang } \\
\text { China }\end{array}$ \\
\hline $\begin{array}{l}\text { Ibrahim Turker } \\
\text { Turkey }\end{array}$ & $\begin{array}{l}\text { Aysegul Verim } \\
\text { Turkey }\end{array}$ & $\begin{array}{l}\text { Meilin Wang } \\
\text { China }\end{array}$ \\
\hline $\begin{array}{l}\text { Tamara Tyrinova } \\
\text { Russian Federation }\end{array}$ & $\begin{array}{l}\text { Satyajeet Verma } \\
\text { India }\end{array}$ & $\begin{array}{l}\text { HaiTao Wang } \\
\text { China }\end{array}$ \\
\hline $\begin{array}{l}\text { Hiroshi Uchinami } \\
\text { Japan }\end{array}$ & $\begin{array}{l}\text { Vaclav Vetvicka } \\
\text { United States of America }\end{array}$ & $\begin{array}{l}\text { Yan Wang } \\
\text { China }\end{array}$ \\
\hline $\begin{array}{l}\text { Alexis Ulrich } \\
\text { Germany }\end{array}$ & $\begin{array}{l}\text { Rafael Vicens } \\
\text { United States of America }\end{array}$ & $\begin{array}{l}\text { Yurong Wang } \\
\text { China }\end{array}$ \\
\hline $\begin{array}{l}\text { Akira Umemura } \\
\text { Japan }\end{array}$ & $\begin{array}{l}\text { Ramon Vilallonga } \\
\text { Spain }\end{array}$ & $\begin{array}{l}\text { Binquan Wang } \\
\text { China }\end{array}$ \\
\hline $\begin{array}{l}\text { Michiaki Unno } \\
\text { Japan }\end{array}$ & $\begin{array}{l}\text { Rene Villadsen } \\
\text { Denmark }\end{array}$ & $\begin{array}{l}\text { Zhong Wang } \\
\text { China }\end{array}$ \\
\hline $\begin{array}{l}\text { Taizen Urahashi } \\
\text { Japan }\end{array}$ & $\begin{array}{l}\text { José-Sebastián Villalón-López } \\
\text { Mexico }\end{array}$ & $\begin{array}{l}\text { Blake Warner } \\
\text { United States of America }\end{array}$ \\
\hline $\begin{array}{l}\text { Jalal Vahedian } \\
\text { Iran }\end{array}$ & $\begin{array}{l}\text { Alessandro Vitale } \\
\text { Italy }\end{array}$ & $\begin{array}{l}\text { Satish Kumar Warrier } \\
\text { Australia }\end{array}$ \\
\hline $\begin{array}{l}\text { J. Fernando Val-Bernal } \\
\text { Spain }\end{array}$ & $\begin{array}{l}\text { Marco Vitellaro } \\
\text { Italy }\end{array}$ & $\begin{array}{l}\text { Jun Watanabe } \\
\text { Japan }\end{array}$ \\
\hline $\begin{array}{l}\text { Katherine Valeros } \\
\text { Singapore }\end{array}$ & $\begin{array}{l}\text { Dimitris Vlachodimitropoulos } \\
\text { Greece }\end{array}$ & $\begin{array}{l}\text { Rafal Watrowski } \\
\text { Germany }\end{array}$ \\
\hline $\begin{array}{l}\text { Sniya Valsa sudhakar } \\
\text { India }\end{array}$ & $\begin{array}{l}\text { My von Euler-Chelpin } \\
\text { Denmark }\end{array}$ & $\begin{array}{l}\text { Jianteng Wei } \\
\text { China }\end{array}$ \\
\hline $\begin{array}{l}\text { Cornelius van de Velde } \\
\text { Netherlands }\end{array}$ & $\begin{array}{l}\text { Christian von Falck } \\
\text { Germany }\end{array}$ & $\begin{array}{l}\text { Helmut Weiss } \\
\text { Austria }\end{array}$ \\
\hline $\begin{array}{l}\text { W.A. van der Zwan } \\
\text { Netherlands }\end{array}$ & $\begin{array}{l}\text { Anna Dorothea Wagner } \\
\text { Switzerland }\end{array}$ & $\begin{array}{l}\text { Bernard Weissman } \\
\text { United States of America }\end{array}$ \\
\hline $\begin{array}{l}\text { Ruud van Erve } \\
\text { Netherlands }\end{array}$ & $\begin{array}{l}\text { Go Wakabayashi } \\
\text { Japan }\end{array}$ & $\begin{array}{l}\text { Annet Westers-Attema } \\
\text { Netherlands }\end{array}$ \\
\hline $\begin{array}{l}\text { Thijs van Oudheusden } \\
\text { Netherlands }\end{array}$ & $\begin{array}{l}\text { Jennifer Waljee } \\
\text { United States of America }\end{array}$ & $\begin{array}{l}\text { Phillip White } \\
\text { United States of America }\end{array}$ \\
\hline $\begin{array}{l}\text { Petr Vavra } \\
\text { Czech Republic }\end{array}$ & $\begin{array}{l}\text { David Wallace } \\
\text { United Kingdom }\end{array}$ & $\begin{array}{l}\text { Andreas Wicki } \\
\text { Switzerland }\end{array}$ \\
\hline $\begin{array}{l}\text { Moniek Vedder } \\
\text { Netherlands }\end{array}$ & $\begin{array}{l}\text { Rohan Walvekar } \\
\text { United States of America }\end{array}$ & $\begin{array}{l}\text { Lisa wiechmann } \\
\text { United States of America }\end{array}$ \\
\hline $\begin{array}{l}\text { Paul Veenboer } \\
\text { Netherlands }\end{array}$ & $\begin{array}{l}\text { Wan Faiziah Wan Abdul Rahman } \\
\text { Malaysia }\end{array}$ & $\begin{array}{l}\text { Sean Williamson } \\
\text { United States of America }\end{array}$ \\
\hline
\end{tabular}


Timothy Wilson

United States Minor Outlying Islands

Andrzej Wincewicz

Poland

Alexander Winter
Germany
Christopher Wong
Australia

Gerald Wright

United States of America

Tao Wu

China

Chuanlong Wu

China

Jian Wu

China

Xinjiang Wu

United States of America

Bin Wu

China

Weiyun Wu

China

Jing Wu

China

Xudong Wu

China

Xing Zhong Wu

China

Jiazeng Xia

China

Wang Xiaoguang

China

Guangqin Xiu

China

Jiejie Xu

China

Fang Xu

China

Yongde $\mathrm{Xu}$

China

Zhi-Xiang Xu

United States of America

Sun Xuecheng

China
Zhu Xulong

China

Daisuke Yabe

Japan

Zhang Yabing

China

Toshiki Yajima

Japan

Kazutaka Yamada

Japan

Sohsuke Yamada

Japan

Tomohiro Yamada

Japan

Takashi Yamada

Japan

Hidetaka Yamamoto

Japan

Kazuyoshi Yamamoto

Japan

Kazuhiro Yamanoi

Japan

Hiroharu Yamashita

Japan

Koji Yamashita

Japan

Ken Yamaura

Japan

Dongrong Yang

China

San-Nan Yang

Taiwan

Zhonghan Yang

China

Haihua Yang

China

Tomoyuki Yano

Japan

Masaahiko Yano

Japan

Katharine Yao

United States of America

Hongtao Ye

United Kingdom
Hui-Ling Yeh

Taiwan

Chun-Nan Yeh

Taiwan

Serkan Yener

Turkey

Fahri Yetisir

Turkey

Cheng-Har Yip

Malaysia

Mitsuhiro Yoneda

United States of America

Tuck Yean Yong

Australia

Zheng Yong

China

Zheng Yong

China

Zheng Yong

China

Kazuhiro Yoshida

Japan

Takaki Yoshikawa

Japan

Yasuo Yoshimura

Japan

Tateki Yoshino

Japan

Hironori Yoshiyama

Japan

Zongbing You

United States of America

Christopher Young

Australia

Qiujun Yu

China

Pengfei Yu

China

Zhi-Gang Yu

China

Wu Yu Jen

Taiwan

Zhong-Yu Yuan

China 
Takeshi Yuasa

Japan

Sumbul Zaheer

Singapore

Nicola Zampieri

Italy

Massoumeh Zargaran

Iran

Nick Zavras

Greece

Bernhard Zelger

Austria

Alessandro Zerbi

Italy

Arife Zeybek

Turkey

Qiang Zhan

China

Xu Zhang

China

Chunlin Zhang

China

Hua Zhang

United Kingdom

Jianhua Zhang

United States of America

Jun Zhang

China

Xiu-Feng Zhang

China

Guo-xin Zhang

China

Qu Zhang

United States of America

Xiaoyu Zhang

China

Sen Zhang

China
Suzhan Zhang

China

Teng Zhang

China

Xing-Hua Zhang

China

Gang Zhao

China

Liang Zhao

China

Yu Pei Zhao

China

Hui Zhao

China

Jing Zhao

United States of America

Shuai Zhen

China

Liduan Zheng

China

Hua-chuan Zheng

China

Jian-yong Zheng

China

Yadong Zheng

China

Shusen Zheng

China

Song Zhengbo

China

Qinghua Zhou

China

Lijun Zhou

China

Li Zhou

China

Weidong Zhou

United States of America
Zhuwei Zhou

China

Tong Zhou

China

Shui-Hong

Zhou

China

Zhi-Xiang Zhou

China

Hua Zhu

United States of America

Xueqiong Zhu

China

Rafal Zielinski

Poland

Vincent Zimmer

Germany

Ouliana Ziouzenkova

United States of America

Panagiotis Zis

Greece

Carmine Zoccali

Italy

Xiangyun Zong

China

Murat Zor

Turkey

Massimiliano Zuccaro

Italy

Changjing Zuo

China

Li Zuo

United States of America 